\title{
Hand Motion Tracking for Alphabet Recognition using
} ANN

\author{
Shivganga Udhan \\ ME student, Computer \\ Department, Sinhgad COE, \\ University of Pune
}

\author{
P. R. Futane \\ Professor \& Head, Computer \\ Dept, Sinhgad COE, University
}

\author{
R. V. Dharaskar \\ Professor \& Director, \\ Matoshree Pratishthan, \\ S.R.T.M. University, Nanded
}

\begin{abstract}
With the Objective of making communication process easy between handicap people and computer this paper describes a very basic method for recognizing the alphabets from hand motion trajectory. This method uses Artificial Neural Network (ANN) for alphabet recognition from gesture path (motion trajectory). This is done in three main stages; preprocessing on video input, feature extraction and classification. In first stage, preprocessing, sticker of particular colour placed on users hand is detected using color information. After detection of required colour to be traced, its motion trajectory which is also called as gesture path will be determined by tracking the sticker placed on hand. In the second stage, features are extracted from hand motion track which can be further used for training \& testing ANN. In the final stage alphabet is recognized by using extracted features of gesture path.
\end{abstract}

\section{General Terms}

Motion Tracking, Human Computer Interaction, Pattern Recognition, Hand Written Character Recognition, Security and Algorithms.

\section{Keywords}

Hand Motion Tracking (HMT), Artificial Neural Network (ANN) and Character Recognition.

\section{INTRODUCTION}

Communication is a major part of human life and is a social activity. Basically, communication is a transfer of information. Based on the channels used for communicating, the communication process can be broadly classified as verbal communication and non-verbal communication. Communication without words is called as Nonverbal communication. And the verbal communication includes written and oral communication. Sending written message is formal way of communication and is original form of the message.

Verbal communication is very advantageous in the situation where speech commands are disturbing, environment is noisy, quantitative information is to be communicated etc. Humancomputer interaction is also one of the emerging field which uses form of verbal communication to make the human interact with computer as they interact with each other. Also there can be physiological impairments like physical disorders, which make writing impossible. But written communication is must in many cases.

To bridge up the communication gap between normal people and physically handicapped people, and also between physically handicapped people and computer, there is requirement of some system which make it possible to have formal written communication by physically handicapped person with other people or computer. Nowadays many systems are working with vision based approach for human motion tracking, but very few work is been done to have formal communication without any physical input device. So, there is the need of a system that can accept the video of human hand or any body part as an input trace out the path $\&$ process it to identify the alphabet from that motion track. Further, it should display a corresponding capital alphabet.

\section{RELATED WORK}

An overview of feature extraction methods for off-line recognition of segmented (isolated) characters is represented by Oivind Due Trier et.al [5].

Vinita Datt and Sunil Datt have proposed a system capable of recognizing handwritten characters or symbols; they build a system that recognizes handwritten characters. The system should be such that it should be able to handle transformation of translation, scaling or a combination of both. The objective of this is to bring out accurate results even for images with noise in them. [9]

M Elmezain, et.al, has presented a method to recognize the alphabets from a single hand motion using Hidden Markov Models (HMM) [3].

A simplified approach to recognition of optical or visual characters is portrayed and discussed by Shashank Araokar [6].

Nisha Vasudeva et.al, have introduced a method in which each image character is comprised of $30 \times 20$ pixels. Features extracted from characters are directions of pixels with respect to their neighbouring pixels. These inputs are given to a back propagation neural network with hidden layer and output layer. They have used the Back propagation Neural Network for efficient recognition where the errors were corrected through back propagation and rectified neuron values were transmitted by feed-forward method in the neural network of multiple layers. [4]

Kauleshwar Prasad, et.al, focused on recognition of English alphabet in a given scanned text document with the help of Neural Networks. Using Matlab Neural Network toolbox, also they have attempted to recognize handwritten characters by projecting them on different sized grids. They have used character extraction and edge detection algorithm for training the neural network to classify and recognize the handwritten characters. [2]

Jianbo Shi and Carlo Tomasi have proposed a feature selection criterion that chooses the correct features which will give optimal use of those features. It is based on how the tracker works, and features that do not correspond to points in the world are also identified. The algorithm used is extension to the previous Newton-Raphson style search methods to work under affine image transformations [1]. 
Te'ofilo E. de Campos et.al, have introduced an approach to incorporate models of the robot-object interaction into the tracking algorithm to effectively improve the performance of the tracker. They first presented the integration of a single robot behavioral model with multiple actions into dynamic Bayesian probabilistic tracking algorithm. So that multi-robot coordination or multi-robot communication can be achieved [8].

Yang Gu and Manuela Veloso, have represented the method that tackles the problem of recognizing characters in images of natural scenes [10]

\section{PROPOSED METHOD}

\subsection{Overview}

Our system can be divided into three main parts; preprocessing of video, feature extraction and classification. The overview of the system is given in following figure1.

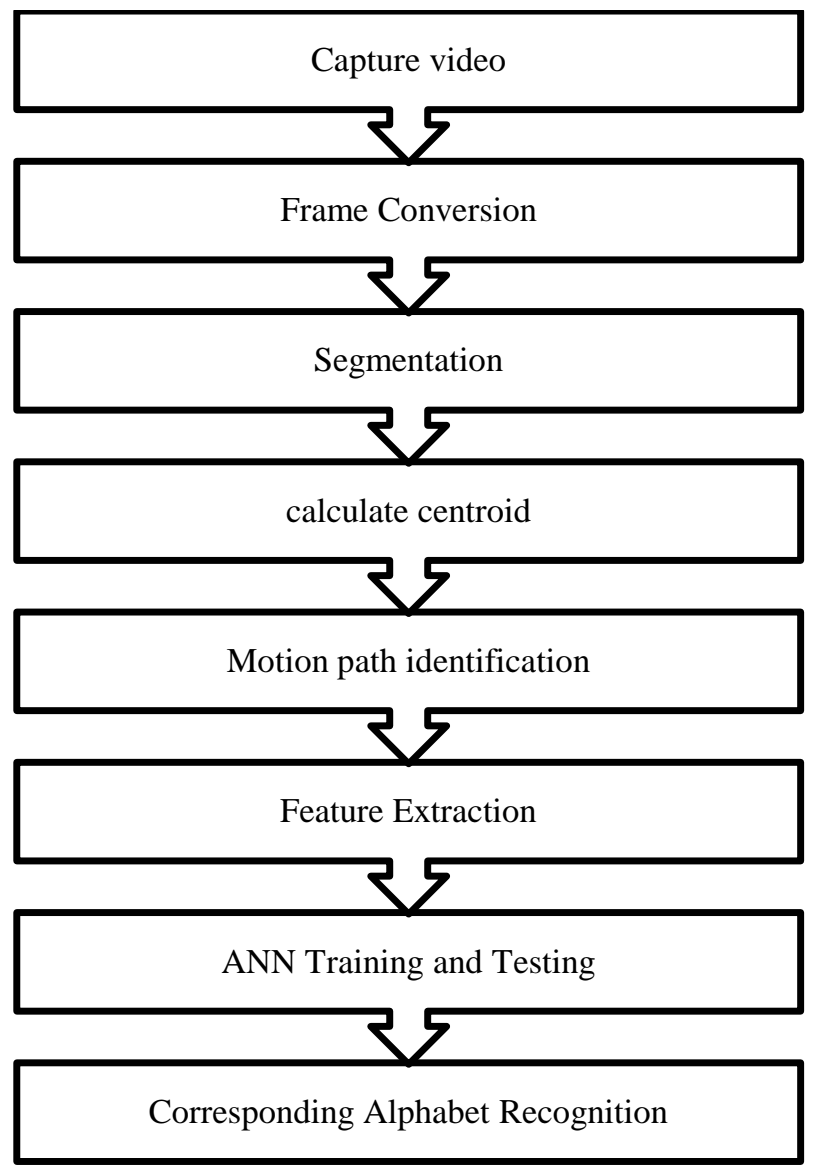

Figure: 1 System Overview.

\subsubsection{Preprocessing}

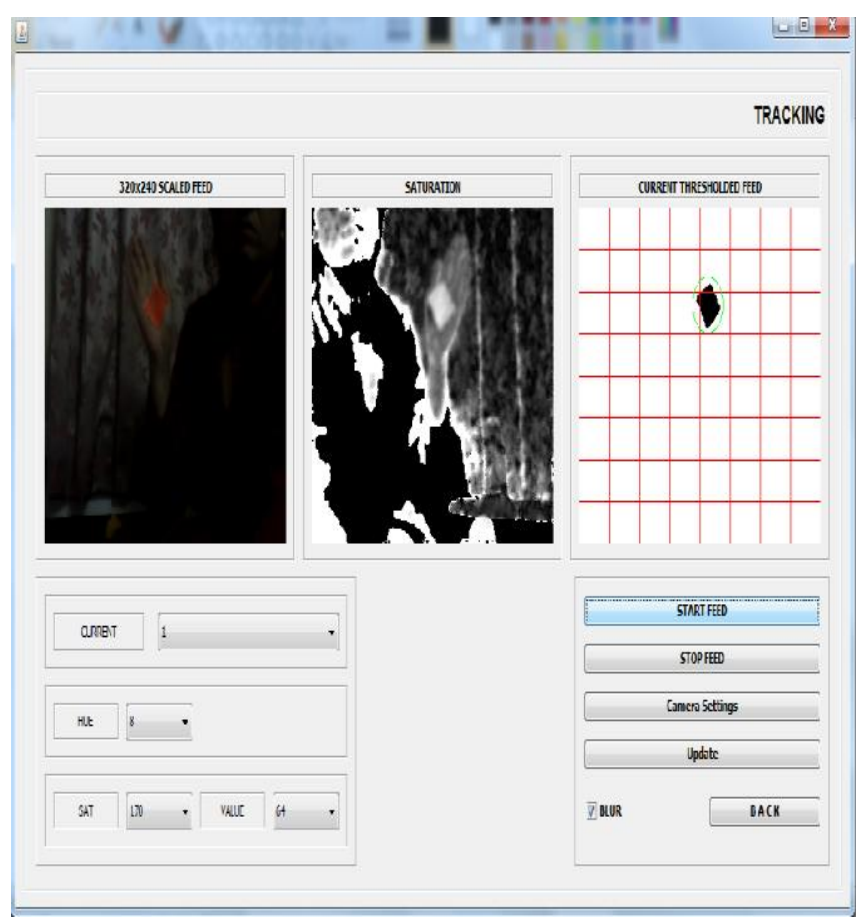

Figure 2: Tracking particular color.

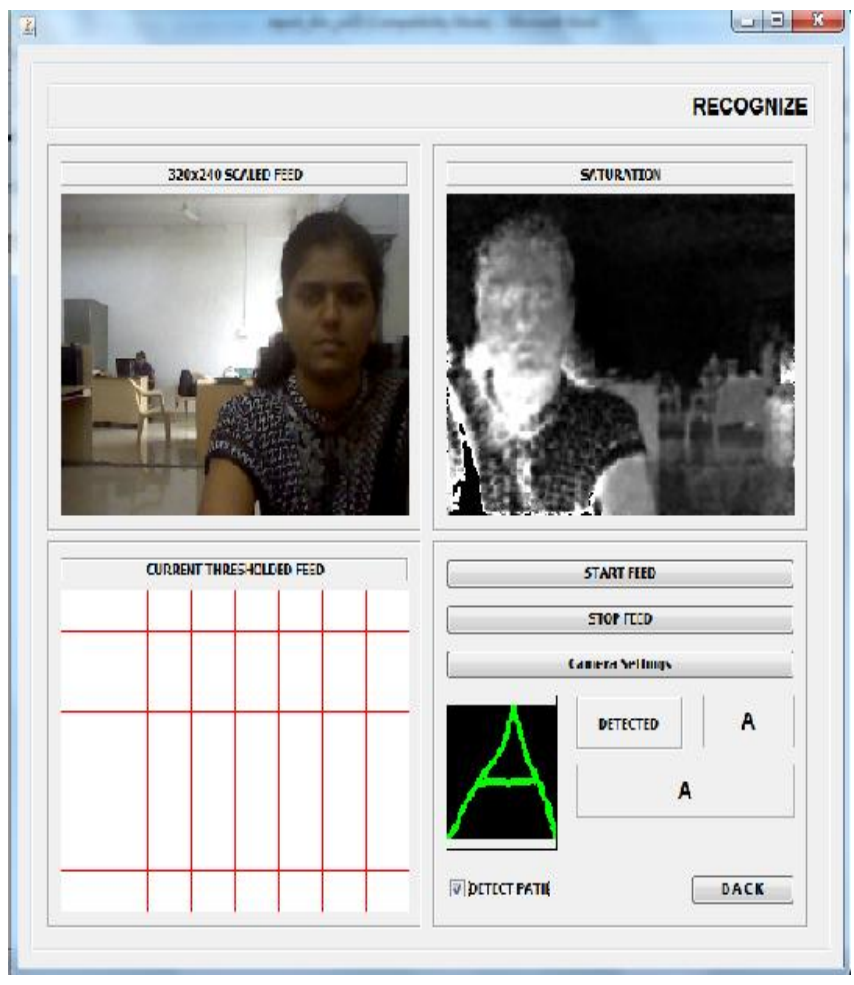

Figure 3: Testing current tracks for an alphabet ' $A$ '.

The system first takes the feed from camera to capture the video. Then user has to manually select the specific color to be traced (particularly a sticker placed on hand). Now the systems interest is to trace the movement of the selected color, for this divide the video in no of frames. For each frame color segmentation is done. Required color in which user is interested in is taken into consideration and its centroid is 
calculated. And lastly the entire centroids together forms path which will be called as hand motion track because sticker is placed on hand.

\subsubsection{Feature Extraction}

The system is finding the feature point matrix of the hand motion track which is shown in figure given below. Images of all the tracks are stored in the database. Now while the time of testing any track the system first finds its feature point matrix and check for a match in database for similar kind of feature point matrix.

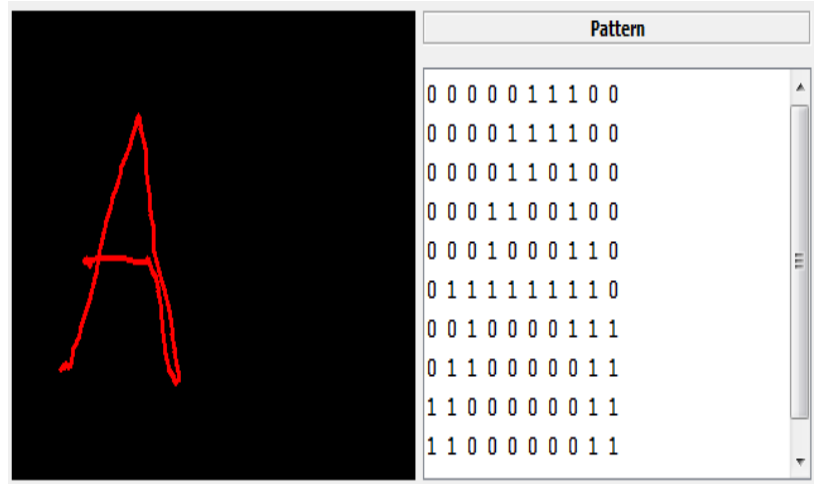

Figure 4: Matching track from database for current track.

Here the $10 \times 10$ feature point matrix is given as input to the neural network. There are total 100 feature points of each character. When system trains multiple samples of same character then the similar cells weight will be increased by 6 . Comparing With: A

\begin{tabular}{|c|c|c|c|c|c|c|c|c|c|c|c|c|c|c|c|c|c|c|c|}
\hline $0<>0$ & $(+0)$ & $0<>0$ & $(+0)$ & $0<>0$ & $(+0)$ & $0<>0$ & $(+0)$ & $0<>0$ & $(+0)$ & $1<>1$ & $(+6)$ & $1<>1$ & $(+6)$ & $1<>0$ & $(-3)$ & $0<>0$ & $(+0)$ & $0<>0$ & $(+0)$ \\
\hline $0<>0$ & $(+0)$ & $0<>0$ & $(+0)$ & $0<>0$ & $(+0)$ & $0<>0$ & $(+0)$ & $1<>0$ & $(-3)$ & $1<>1$ & $(+6)$ & $1<>1$ & $(+6)$ & $1<>1$ & $(+6)$ & $0<>0$ & $(+0)$ & $0<>0$ & $(+0)$ \\
\hline $0<>0$ & $(+0)$ & $0<>0$ & $(+0)$ & $0<>0$ & $(+0)$ & $0<>0$ & $(+0)$ & $1<>0$ & $(-3)$ & $1<>1$ & $(+6)$ & $0<>1$ & $(-3)$ & $1<>1$ & $(+6)$ & $0<>0$ & $(+0)$ & $0<>0$ & $(+0)$ \\
\hline $0<>0$ & $(+0)$ & $0<>0$ & $(+0)$ & $0<>0$ & $(+0)$ & $1<>0$ & $(-3)$ & $1<>1$ & $(+6)$ & $0<>1$ & $(-3)$ & $0<>0$ & $(+0)$ & $1<>1$ & $(+6)$ & $0<>0$ & $(+0)$ & $0<>0$ & $(+0)$ \\
\hline $0<>0$ & $(+0)$ & $0<>0$ & $(+0)$ & $0<>0$ & $(+0)$ & $1<>1$ & $(+6)$ & $0<>1$ & $(-3)$ & $0<>0$ & $(+0)$ & $0<>0$ & $(+0)$ & $1<>1$ & $(+6)$ & $1<>1$ & $(+6)$ & $0<>0$ & $(+0)$ \\
\hline $0<>0$ & $(+0)$ & $1<>0$ & $(-3)$ & $1<>1$ & $(+6)$ & $1<>1$ & $(+6)$ & $1<>1$ & $(+6)$ & $1<>0$ & $(-3)$ & $1<>0$ & $(-3)$ & $1<>1$ & $(+6)$ & $1<>1$ & $(+6)$ & $0<>0$ & $(+0)$ \\
\hline $0<>0$ & $(+0)$ & $0<>1$ & $(-3)$ & $1<>1$ & $(+6)$ & $0<>0$ & $(+0)$ & $0<>0$ & $(+0)$ & $0<>0$ & $(+0)$ & $0<>0$ & $(+0)$ & $1<>1$ & $(+6)$ & $1<>1$ & $(+6)$ & $1<>0$ & $(-3)$ \\
\hline $0<>0$ & $(+0)$ & $1<>1$ & $(+6)$ & $1<>1$ & $(+6)$ & $0<>1$ & $(-3)$ & $0<>1$ & $(-3)$ & $0<>1$ & $(-3)$ & $0<>1$ & $(-3)$ & $0<>1$ & $(-3)$ & $1<>1$ & $(+6)$ & $1<>1$ & $(+6)$ \\
\hline $1<>1$ & $(+6)$ & $1<>1$ & $(+6)$ & $0<>1$ & $(-3)$ & $0<>1$ & $(-3)$ & $0<>1$ & $(-3)$ & $0<>1$ & $(-3)$ & $0<>1$ & $(-3)$ & $0<>1$ & $(-3)$ & $1<>1$ & $(+6)$ & $1<>1$ & $(+6)$ \\
\hline $1<>1$ & $(+6)$ & $1<>1$ & $(+6)$ & $0<>1$ & $(-3)$ & $0<>0$ & $(+0)$ & $0<>0$ & $(+0)$ & $0<>0$ & $(+0)$ & $0<>0$ & $(+0)$ & $0<>0$ & $(+0)$ & $1<>1$ & $(+6)$ & $1<>1$ & $(+6)$ \\
\hline
\end{tabular}

Figure 6: Score when comparing A's track with A.

\begin{tabular}{|c|c|c|c|c|c|c|c|c|c|c|c|c|c|c|c|c|c|c|c|}
\hline $0<>0$ & $(+0)$ & $1<>0$ & $(-3)$ & $1<>0$ & $(-3)$ & $1<>0$ & $(-3)$ & $1<>0$ & $(-3)$ & $1<>1$ & $(+6)$ & $1<>1$ & $(+6)$ & $1<>0$ & $(-3)$ & $1<>0$ & $(-3)$ & $1<>0$ & $(-3)$ \\
\hline $1<>0$ & $(-3)$ & $1<>0$ & $(-3)$ & $0<>0$ & $(+0)$ & $0<>0$ & $(+0)$ & $0<>0$ & $(+0)$ & $0<>1$ & $(-3)$ & $0<>1$ & $(-3)$ & $0<>1$ & $(-3)$ & $0<>0$ & $(+0)$ & $1<>0$ & $(-3)$ \\
\hline $1<>0$ & $(-3)$ & $0<>0$ & $(+0)$ & $0<>0$ & $(+0)$ & $0<>0$ & $(+0)$ & $0<>0$ & $(+0)$ & $0<>1$ & $(-3)$ & $0<>1$ & $(-3)$ & $0<>1$ & $(-3)$ & $1<>0$ & $(-3)$ & $1<>0$ & $(-3)$ \\
\hline $1<>0$ & $(-3)$ & $1<>0$ & $(-3)$ & $0<>0$ & $(+0)$ & $0<>0$ & $(+0)$ & $0<>1$ & $(-3)$ & $1<>1$ & $(+6)$ & $1<>0$ & $(-3)$ & $1<>1$ & $(+6)$ & $1<>0$ & $(-3)$ & $1<>0$ & $(-3)$ \\
\hline $1<>0$ & $(-3)$ & $1<>0$ & $(-3)$ & $1<>0$ & $(-3)$ & $1<>1$ & $(+6)$ & $1<>1$ & $(+6)$ & $1<>0$ & $(-3)$ & $1<>0$ & $(-3)$ & $0<>1$ & $(-3)$ & $0<>1$ & $(-3)$ & $0<>0$ & $(+0)$ \\
\hline $1<>0$ & $(-3)$ & $1<>0$ & $(-3)$ & $1<>1$ & $(+6)$ & $1<>1$ & $(+6)$ & $1<>1$ & $(+6)$ & $1<>0$ & $(-3)$ & $0<>0$ & $(+0)$ & $0<>1$ & $(-3)$ & $0<>1$ & $(-3)$ & $0<>0$ & $(+0)$ \\
\hline $1<>0$ & $(-3)$ & $0<>1$ & $(-3)$ & $0<>1$ & $(-3)$ & $0<>0$ & $(+0)$ & $0<>0$ & $(+0)$ & $1<>0$ & $(-3)$ & $1<>0$ & $(-3)$ & $0<>1$ & $(-3)$ & $0<>1$ & $(-3)$ & $0<>0$ & $(+0)$ \\
\hline $1<>0$ & $(-3)$ & $0<>1$ & $(-3)$ & $0<>1$ & $(-3)$ & $0<>1$ & $(-3)$ & $0<>1$ & $(-3)$ & $0<>1$ & $(-3)$ & $1<>1$ & $(+6)$ & $1<>1$ & $(+6)$ & $0<>1$ & $(-3)$ & $0<>1$ & $(-3)$ \\
\hline $1<>1$ & $(+6)$ & $0<>1$ & $(-3)$ & $0<>1$ & $(-3)$ & $0<>1$ & $(-3)$ & $0<>1$ & $(-3)$ & $1<>1$ & $(+6)$ & $1<>1$ & $(+6)$ & $0<>1$ & $(-3)$ & $0<>1$ & $(-3)$ & $0<>1$ & $(-3)$ \\
\hline $1<>1$ & $(+6)$ & $1<>1$ & $(+6)$ & $1<>1$ & $(+6)$ & $1<>0$ & $(-3)$ & $1<>0$ & $(-3)$ & $1<>0$ & $(-3)$ & $1<>0$ & $(-3)$ & $0<>0$ & $(+0)$ & $0<>1$ & $(-3)$ & $0<>1$ & $(-3)$ \\
\hline
\end{tabular}

Figure 7: Score when comparing A's track with B.

The process of recognition can be clearer by above figures. Track of current character is matched with all tracks available in the database. Above track is more matching with trained A with following track and feature point matrix.
There will be some cells matching with non-similar characters then the weight of that cell will get reduced by 3 .

At the time of testing current feature point matrix is compared with all the weight matrix of the trained characters. If match found for existing non-zero points then add value 6 to the score else subtract value 3 from the score. Finally add all these values to get the final score or weight of that particular character. This process is shown in following series of figures. Now this weight is compared with the weights of trained characters. Then the character whose weight is more near to the current weight is displayed as an output.
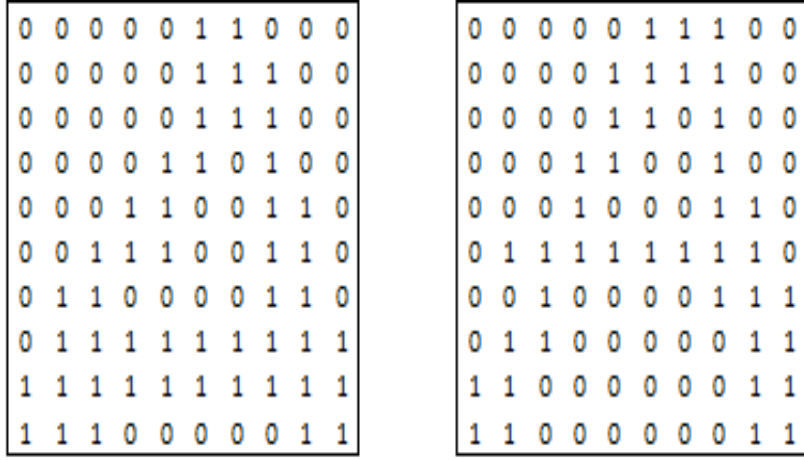

Figure: 5 Feature point matrix of (a) Current track (b) Matching track from trained data.

\subsubsection{Classification}

Feed Forward Artificial Neural Network (FF-ANN) is used for classification as shown in figure given below. 


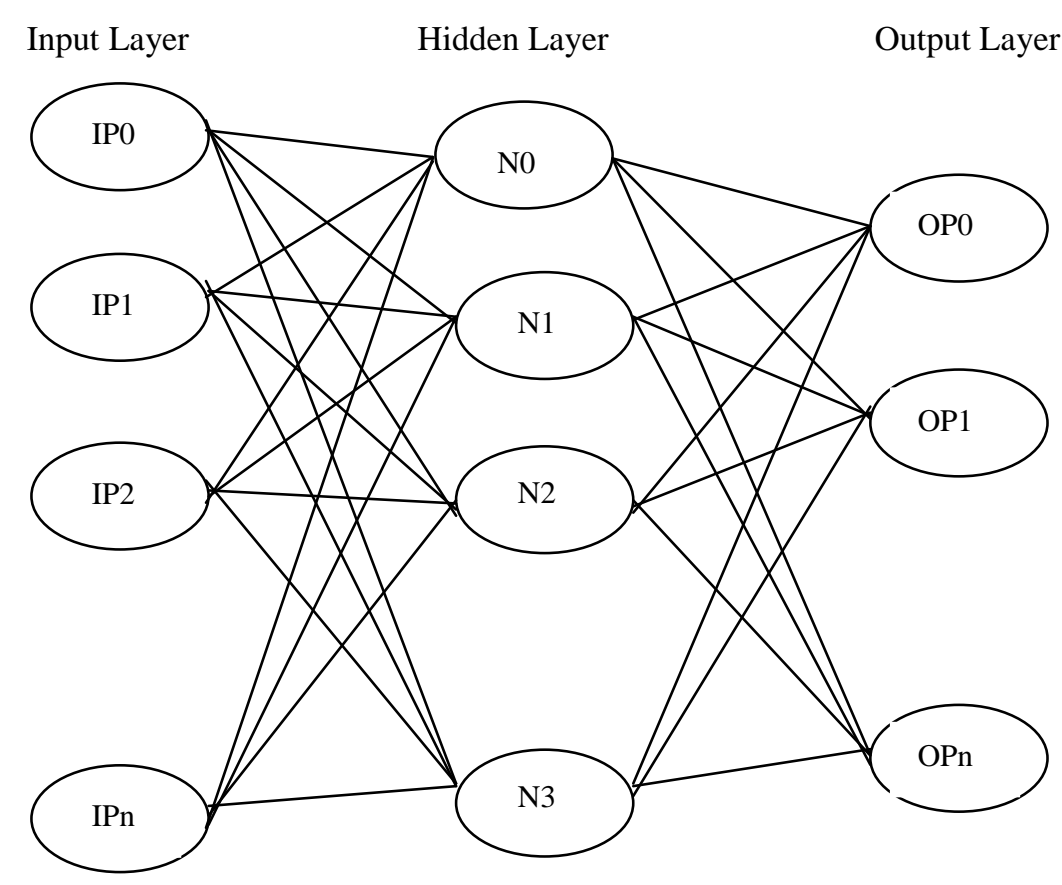

Figure 8: Architecture of FF ANN for HMT system

The system has been using supervised training method, in which it assigns the alphabet for corresponding hand motion track. For e.g. if system have 10 different track of alphabet 'A' then all those tracks will fall under single class called as 'A'. Similarly for each alphabet a separate class is created. At the time of testing once the system has found a HMT, it will search for corresponding class and the most matching track in that class. The track which gives highest score when compared with current track is identified and the corresponding alphabet is displayed.

\subsubsection{Algorithm for Training \\ 1) START}

2) Accept the input video.

3) Extract the frames from input video.

4) For each frame

i) Point out the selected color in frame;

ii) Calculate Centroid;

iii) End.

5) Find the Hand Motion Track (HMT) from all centroids.

6) Calculate the Features from (HMT);

7) Save the image of track to Database

8) Classify Tracks

9) STOP.

\subsubsection{Algorithm for Testing}

1) START
2) Accept the input video.

3) Extract the frames from input video.

4) For each frame

i) Point out the selected color in frame;

ii) Calculate Centroid;

iii) End.

5) Find the Hand Motion Track (HMT) from all centroids.

6) Calculate the Features from (HMT);

7) Map the track with database.

If Track is mapped

i) Identify the corresponding alphabet.

ii) Display that alphabet.

Else

Discard Track.

9) STOP

\section{RESULT AND DISSCUSSION}

The system is working for runtime videos. The data set of 7 tracks for each of 26 alphabets i.e. total 182 tracks is used. All the 26 alphabets are trained and tested to get the accuracy of about $95 \%$ for users familiar with the system. User can train numbers and other characters like devnagri letters and check for its recognition using this system. This means that the system can be made language independent in future. Thus this system gives very easy but effective method for real time motion tracking and alphabet recognition. 


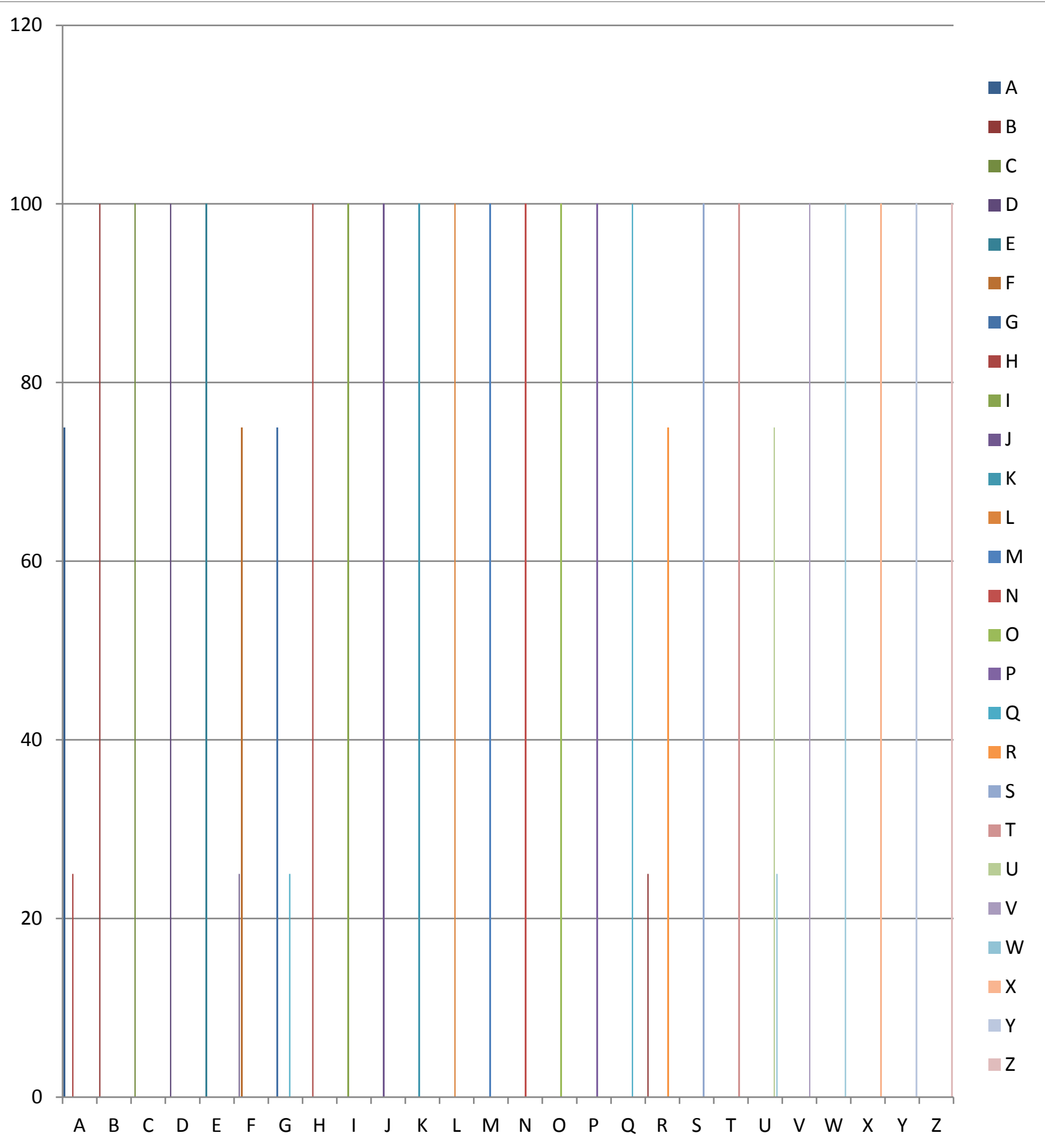

Figure 9: Recognition Result for all Alphabets. 
Table 1. Table captions should be placed above the table

\begin{tabular}{|l|l|l|}
\hline Features & $\begin{array}{l}\text { ANN Based } \\
\text { Method }\end{array}$ & $\begin{array}{l}\text { Distance Based } \\
\text { Method [7]. }\end{array}$ \\
\hline $\begin{array}{l}\text { No. of characters } \\
\text { recognized }\end{array}$ & All 26 & 22 \\
\hline $\begin{array}{l}\text { Mode of } \\
\text { operation }\end{array}$ & Online & Offline \\
\hline Complexity & Low & High \\
\hline Accuracy & $95 \%$ & $85 \%$ \\
\hline
\end{tabular}

\section{CONCLUSION}

An attempt is made to develop a Hand Motion Tracking system which is scale and translation invariant using ANN approach. It is possible to process videos at real time. As system is using supervised training algorithm it is possible to have a language independent system which will be able to recognize devnagri characters or any other language characters. System can be enhanced to include all possible characters of any language. In future multiple words can be written to form a sentences and the paragraph.

\section{REFERENCES}

[1] Jianbo Shi and Carlo Tomasi, "Good Features to Track", IEEE, National science Foundation, 1994, pp. 593-601.

[2] Kauleshwar Prasad, et.al., "Character Recognition Using Matlab's Neural Network Toolbox", International Journal of $u$ - and e-Service, Science and Technology, Science and Engg. Research Support Center, Vol. 6, No. 1, February, 2013
[3] M Elmezain, et.al, "Gesture Recognition for alphabet from Hand Motion Trajectory Using Hidden Markov Models". IEEE International Symposium on Signal Processing and Information Technology, 2007, pp:11921197.

[4] Nisha Vasudeva, Hem Jyotsana Parashar and Singh Vijendra, "Offline Character Recognition System Using Artificial Neural Network", International Journal of Machine Learning and Computing, Vol. 2, No. 4, August 2012, pp. 449-453

[5] Oivind Due Trier, Anil K. Jain and Torfinn Taxt, "Feature Extraction Methods for Character RecognitionA Survey", Pattern Recognition, Vol. 29, No. 4, 1996, pp. 641-662.

[6] Shashank Araokar, Visual Character Recognition using Artificial Neural Networks.

[7] Shivganga Udhan and Prof. Pravin Futane, "Alphabet Recognition Using Hand Motion Track" International Journal of Engineering Research and Applications (IJERA) ISSN: 2248-9622, Vol. 3, Issue 1, JanuaryFebruary 2013, pp.289-293

[8] Te'ofilo E. de Campos, Bodla Rakesh Babu and Manik Varma, "Character Recognition in Natural Images".

[9] Vinita Dutt and Sunil Dutt, "Hand Written Character Recognition Using Artificial Neural Network", Advances in Computing, Scientific \& Academic Publishing, 2011, pp. 18-23.

[10] Yang Gu and Manuela Veloso, "Effective Multi-Model Motion Tracking using Action Models", The International Journal of Robotics Research, January2009; Vol. 28, No. 3, pp. 3-19. 